\title{
Amputations around the Ankle and Hind-foot
}

\author{
L. P. SEIMON \\ M.B., M.Ch. Orth., F.R.C.S. (Edin.) \\ St. Augustine's Medical Centre, \\ Durban.
}

Of the various amputations performed around the ankle and hind-foot the majority have been discarded because of numerous problems that have been encountered. In fact many standard texts mention some of the procedures, only to condemn them. Amputations distal to the metatarsal bases generally present no problem and will not be considered further in this paper. Those through the tarsometatarsal region (Lisfranc) are seldom performed because of the equinus deformity that is known to occur post-operatively. The Chopart procedure (through the mid-tarsal region) has also been abandoned because of the severe equino-valgus deformity that results due to the unopposed action of the gastrosoleus group. Pirigoff's amputation is one in which the talus is excised, as is most of the calcaneus. The remaining proximal part of the calcaneus is then tilted vertically so that its raw distal surface comes up against the distal end of the tibia. This results in a short stump necessitating a raised prosthesis. Amputation has also been performed through the distal (anterior) end of the calcaneus. The talus is excised and a straightforward fusion between tibia and calcaneus carried out. This too, results in slight shortening, but the patient has a good end-bearing pad which does not deform, thanks to the arthrodesis.

The Syme's amputation (through the distal quarter-inch of tibia, and with suture of the heel pad to cover this raw surface) remains a well-tried and reliable operation. Unforunately, it too results in a short stump and the prosthesis that is fitted is somewhat bulky and generally unacceptable to most female patients.

For various reasons, therefore, many surgeons have been inclined to abandon amputations proximal to the metatarsal bases and distal to the Syme's level. An objective of this paper is to make one reconsider these procedures in the light of some minor modifications that I have found to be most gratifying. No one can deny the tremendous benefits to a patient of having an end-bearing stump that he can get about on, even without the aid of a prosthesis. Modern prostheses have also been improved considerably and are not as bulky as they were previously. For many of our patients, notably the lepers, cosmesis is unimportant. The overriding consideration is to enable them to walk again. It has been generally accepted that a solution to the salvage of the grossly deformed foot in these patients is to perform a below-knee amputation. The patient is then dependent on a pylon and frequently has to use crutches or a cane as well. Some unfortunates have no fingers (due to absorption) and cannot manage crutches. Should something go wrong with the pylon, they are, once again, totally incapacitated. The site of large ulcerated areas over pressure points in grossly deformed feet has usually been enough for most surgeons not to give local amputations in the region a second thought. My own experience proves otherwise, and it is gratifying to note similar views to my own expressed in a recent article by Hart, Williams and Scott. ${ }^{1}$

Naturally, should there be some other good reason to contra-indicate amputation through the hind-foot, such as a deficient circulation, then obviously the choice must be to go for a more proximal level.

Ulcerated areas and evidence of chronic osteomyelitis are not contra-indications to surgery, but these areas must be adequately excised as part of the procedure.

In all cases my objectives and approach are:

1. To preserve as much length as possible without jeopardising the chances of survival of the skin flaps. In the mid-tarsal region I would sacrifice some bone on the plantar aspect, rather than dorsally, making the line of the amputation oblique. The little extra length of bone on the dorsum of the foot may make all the difference as to whether the patient requires any prosthesis at all, other than a simple toe-filler in a normal shoe.

2. To anticipate deformity and therefore prevent this by rebalancing the musculature. In tarsometatarsal amputations the long extensor tendons must be transferred into the medial part of the cuboid or the lateral cuneiform. This will balance the foot beautifully, and together with the action of the tibialis anterior, will prevent the occurrence of an equinus deformity. Should there be weakness of the toe extensors, then a tibialis posterior transfer through the interosseus membrane is performed. Where the Achilles' tendon is already contracted, it is lengthened. The Chopart's (mid-tarsal) amputation is modified by transferring the tibialis anterior and extensor digitorum longus tendons into the neck of the talus. This adequately counters the gastrosoleus action.

3. To give the patient a sturdy end-bearing stump that will stand up to the ravages of daily usage, it is imperative that the weight-bearing surface is normal plantar skin. The ulceration in case (4) is largely due to the fact that, because of the severe equinus deformity, dorsal skin has become the weight-bearing area. In order to get plantar skin back into an end-bearing position, the procedure must be modified according to the particular case. This is best illustrated by referring to cases (3), (4) and (5). In order to maintain the hee] pad in position, I prefer not to excise the os calcis completely as this disrupts the firm union that the pad has to that bone. It is much more practicable to preserve as much of the os calcis as possible or even a sliver, if most has to be sacrificed, and to get this to fuse to the distal end of the tibia. Bone heals to bone far better than soft tissue adheres to it. Depending on the dictates of the particular case, either a modified Syme's Procedure (retaining a thin piece of os calcis), or a Pirigoff or straightforward tibio-calcaneal fusion is carried out. When sufficient os calcis is left the fusion is facilitated by using a simple compression device. In the modified Syme's Procedure, a Steinman's Pin passed up through the sole into the tibia will hold the position while fusion occurs.

4. To completely excise any ulcer and/or infected bone that is present. Generally the ulcer has resulted due to pressure over a bony prominence. In almost all cases the ulcer has to be excised together with a large underlying wedge of bone, the latter fortunately also helps to correct the defornity.

\section{Results}

I have been astounded at how well some of the grossly infected leprosy cases have healed. These patients are usually fitted with a Jumbo boot, but most of them are able to get about on their stumps without the aid of any prosthesis at all.

When amputation is perforned at the tarsometatarsal region, so that a small tongue of stump projects beyond the line of the anterior margin of the shin, the patients can frequently get by without having to use any prosthesis at all. I usually recommend a Velskoen-type shoe with a simple sponge rubber toe-filler. 'Breaking' of the shoe at the tarsometatarsal level has not been a problem. When it is, then simple reinforcing of the sole is usually all that is necessary. 
It must be remembered that amputation at a higher lctel can always be carried out later, should the primary procedurc fail. Thus far, only two cases have required this. Or these, one patient requested a below-knee amputation, so that he could be fitted with a limb to match the prosthesis on his other leg.

\section{ILIUSTRATIVF: CASES}

\section{Case No. 1:}

T.B.J., age 33 years.

This patient was suffering from peroneal musculal dystrophy. He developed bilateral pes cavus and seven years prior to being seen by me he had had operations perlormed on both his feet. The results were cxtlemely unsatisfactory and the patient walked with greal difficulty and had a tremendous amount of pain. Rigid clawing or the toes was present with multiple callosities over pressure areas, the right being much worse than the left. A triple arthrodesis had also been performed on the left and this was satisfactory. The soft tissues of the distal hall of the right foot nere markedly fibrotic and atrophic, and he also had a fixed equinus deformity. He was able to walk with only the utmost difficulty, due both to the pain and the deformitics.

All the toes of the left foot were amputated. On the right amputation was carricd out through the distal hall of the cuneiforms. The E.D.L. tendons were transferred to the lateral cuneiform and the Achilles tendon was elongated. It is now two and a half years since the operation and the patient has done extremely wcll. He wears ordinary shoes with toe-filler's, has a minimal limp and no pain whatever.

\section{Case No. 2:}

Mrs. R.E., aged 43 years.

This unfortunate lady had nine operations performed on her right foot after a "procedure for hallux valgus had gone wrong".

When seen she was grossly incapaciated by pain, extending from the mid-tarsal region distally. Soft tissues in the arca were extremely fibrotic, and fixed deformities of all the roes were present. Further surgery to correct the toes was out of the question and in order to cradicate her pain an amputation had to be performed. She had several opinions, all of whom suggested a below-knee amputation. Instead I performed an amputation immediately proximal to the larsometatarsal joints and with the bone ends bevelled posteriorly towards the plantar aspect. The insertion of the tibialis anterior was reinforced medially with chromic sutures and the E.D.L. tendons were transferred to the lateral cunciform and cuboid. She has made excellent progress and leads a perfectly normal life. At first, various prostheses were tried, but she now wears ordinary shoes with a simple toe-filler. Her range of movement is well illustrated in figs. 1-6.

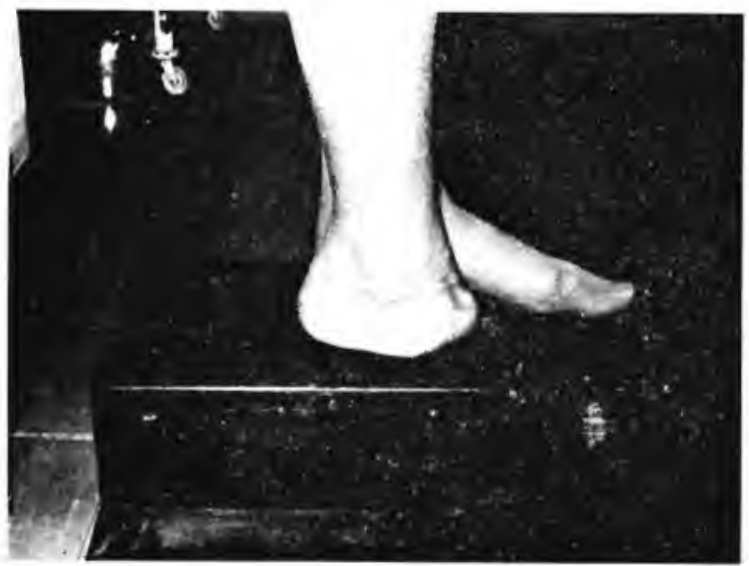

Fig. 1. Case 2: Mrs. R.E. WEIGHT-BEARING (LAT. VIEW). Note slight obliquity of stump, being longer dorsally.

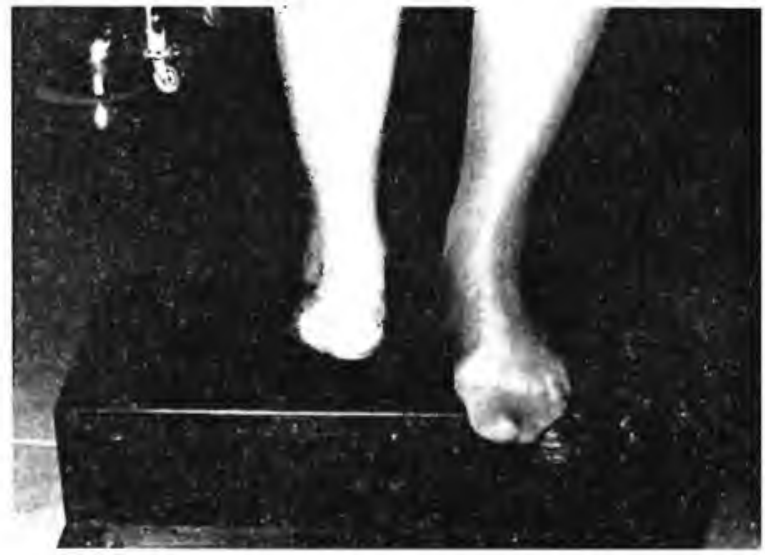

Fig. 2. Case 2: Mrs. R.E. IVEIGHT-BEARING (ANT. VIEW).

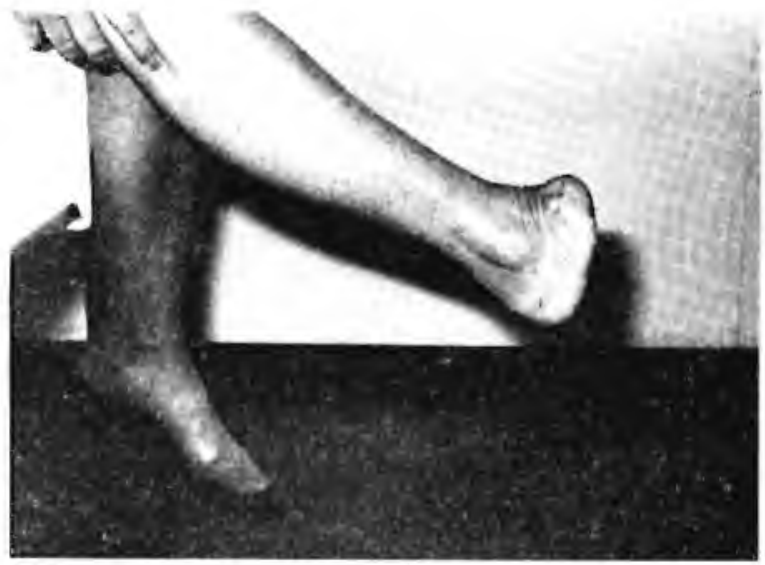

Fig. 3. Case 2: Mrs. R.E. ACTIVE DORSIFLEXION.

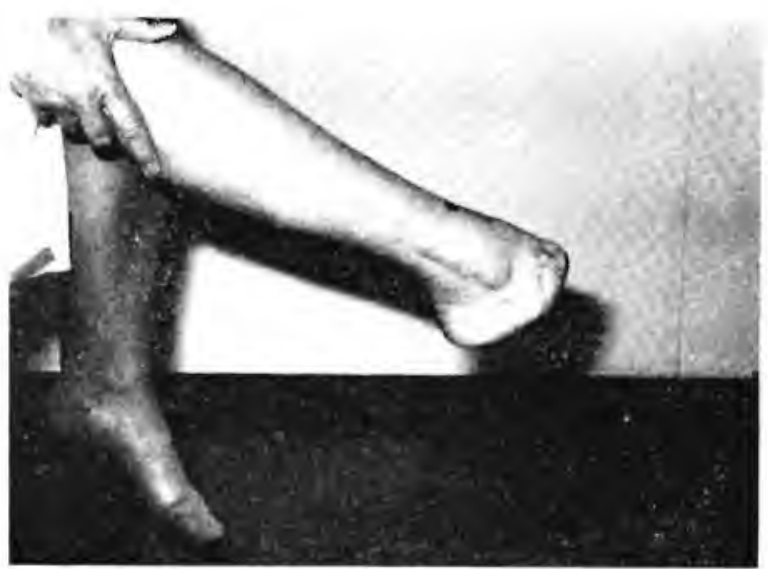

Fig. 4. Case 2: Mrs. R.E. ACTIVE PLANTARFLEXION 


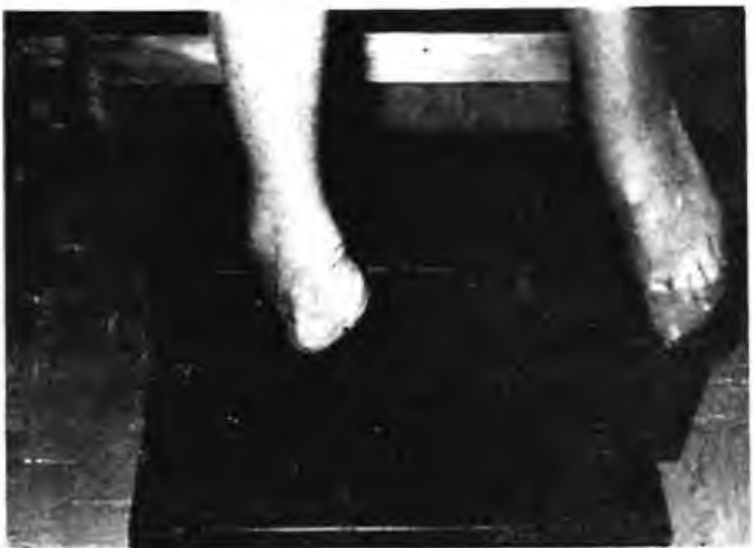

Fig. 5. Case 2: Mrs. R.E. ACTIVE INVERSION.

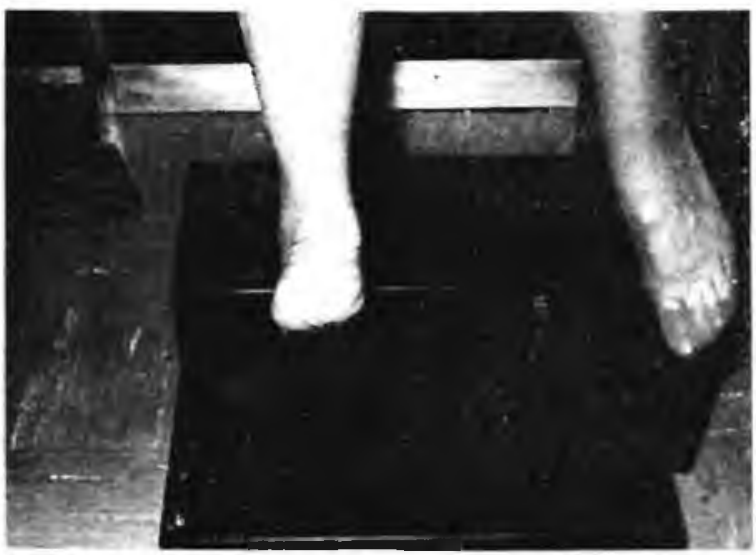

Fig. 6. Case 2: Mrs. R.E. ACTIVE EVERSION

Case No. 3:

M.S., age 36. Leper

As can be seen in Fig. 7 the heel has been pulled into gross equinus and weight is now taken on dorsal skin which covers the distal end of the tibia. A sub-cutaneous Achilles tenotomy was performed and the heel was rotated under the tibia. The opposing bone surfaces were excised and the rau ends held together by means of a compression clamp. The wound became grossly infected but this settled down rapidly with treatment. (Fig. \&.) A good end-bearing stump resulted.

Case No. 4:

S.K., age 55 years, Leper

The appearance of this paticnt's feet can be seen in Figs. 9-11. The large ulcer over the end-bearing area of the left stump did not prevent a primary Syme s type amputation being performed. The ulcer, logether with a wedge of bone was excised, leaving approximately hall an inch thickness of the os calcis. This was fused to the tibia. Healing uas uneventful but unfortunately the resultant heel was in mild varus. This was corrected subsequently by excision of a small laterally based wedge.

On the right side a mid-tarsal amputation was first performed with excision of the lateral ulcer, using a medially based flap to cover the end. A tibio-calcaneal fusion was planned as a second stage to be performed later.

Case No. 5:

N.W., age 58, Leper.

The appearance of the feet can be clearly visualized by referring to Figs. 12 and 13
A wedge of skin and bone was excised from the right foot and the remainder of the calcaneus fused to the tibia, using a compression clamp. This brought the heel pad into its normal weight bearing position. In the left foot a tibiocalcaneal fusion was performed, the superior surface of the os calcis being cut horizontally and held against the tibia by means of a compression clamp. Both uounds healed primarily and excellent weight-bearing stumps resulted.

\section{Case No. 6:}

Mt. S., age 35, Leper.

This patient presented with severe bilateral equino-varus deformities. absorption of the lateral four rays of each foot

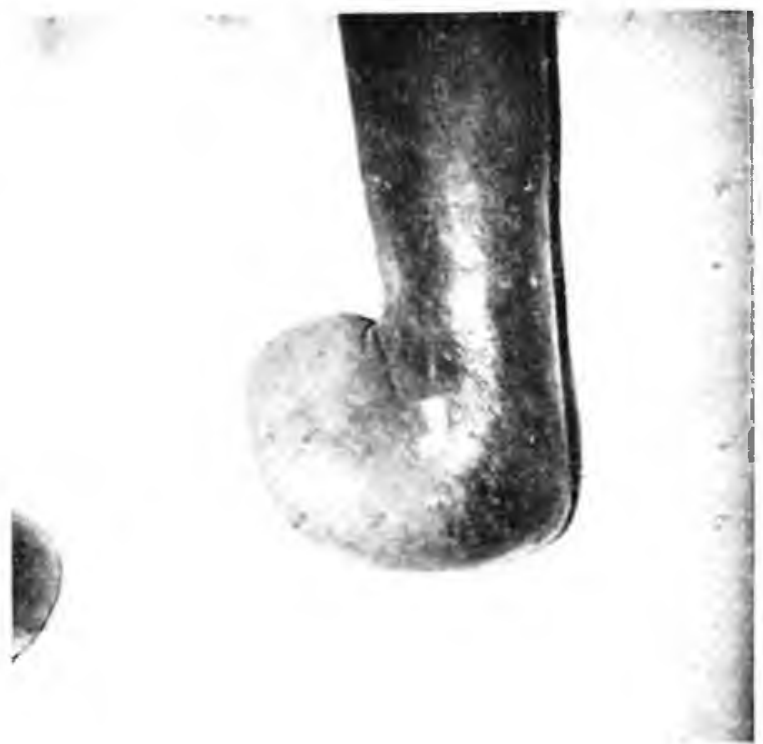

Fig. 7. Case 3: M.S., Leper. LAT. VIEW LEFT FOOT. Note how the calcaneus has been pulled up into gross equinus and lies posterior to the tibia.

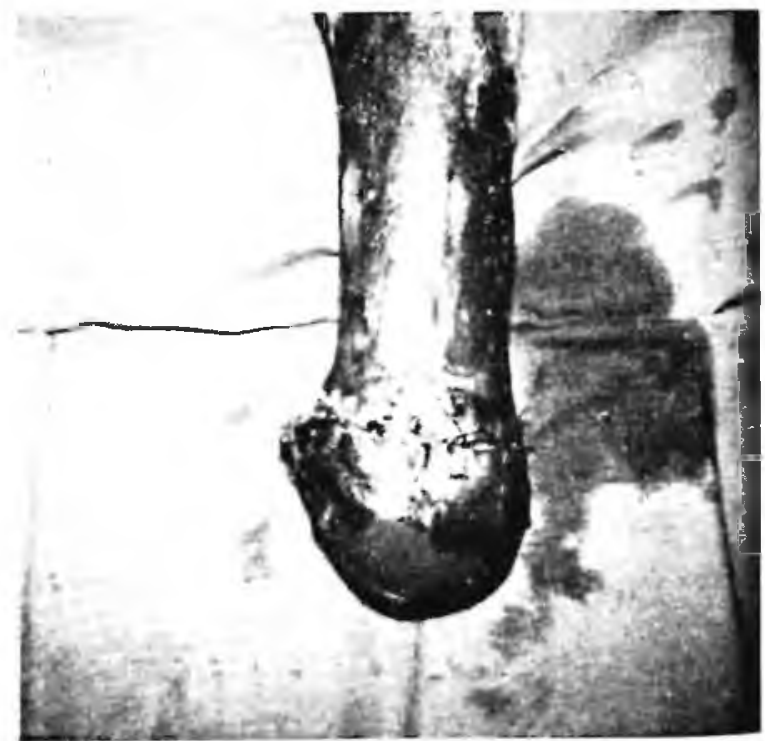

Fig. 8. Case 3: M.S. FOUR WEEKS AFTER TIBIO CALCANEAL FUSION. Compression clamp has just been removed. Note how the heel fat pad has been restored to its normal position for weight-bearing. 
and with overt infection extending deeply into the tali. Despite the infection bilateral Syme's type amputations were performed, with excision of all obviously infected bone. Both stumps healed per primum and the patient ras fitted with surgical stump boois.
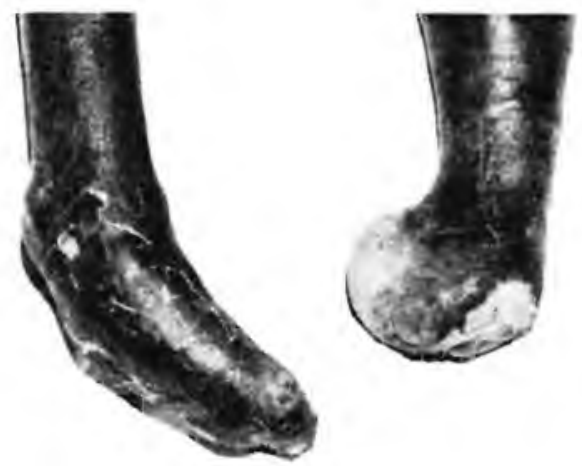

Fig. 9. Case 4: S.K., Leper. The left stump reveals a marked equinus deformity with a large ulcer over the abnormal pressure-bearing area. The equino-varus deformity of the right foot is also depicted.

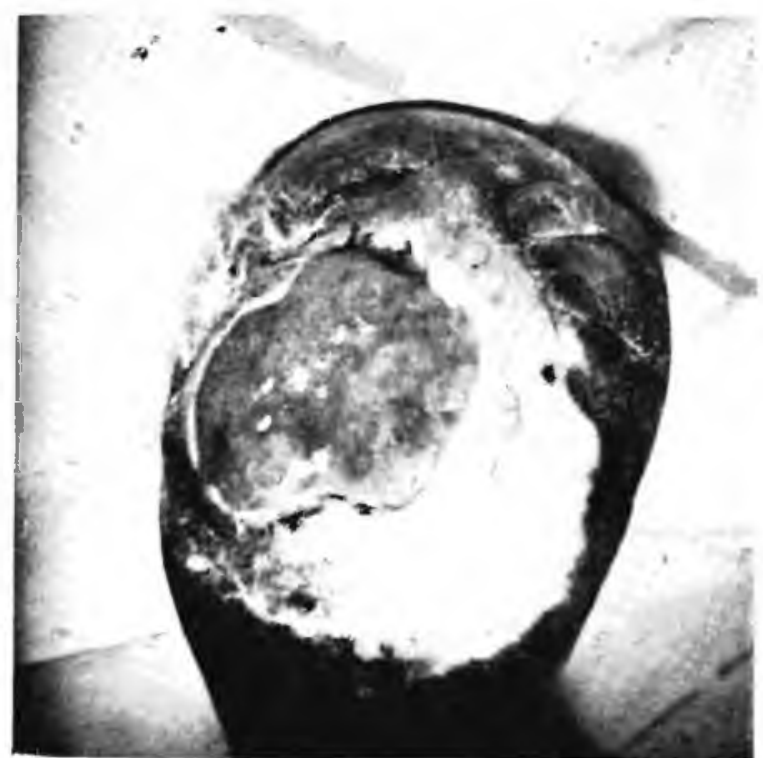

Fig. 10. Case 4: S.K. Showing the extent of the ulceration on the left stump.

\section{SUMMARY}

Illustrative cases reveal that amputation through the hind-foot can result in excellent weight-bearing stumps and that previously expected deformities can be prevented by anticipating them and by transferring tendons accordingly, to rebalance the foot. The presence of ulceration and infection does not preclude this type of surgery.

\section{REFERENCE}

1. Hart, R. J., Williams, H. W., and Scott, G. R. Leprosy Review, 40, No. 1, 59 .

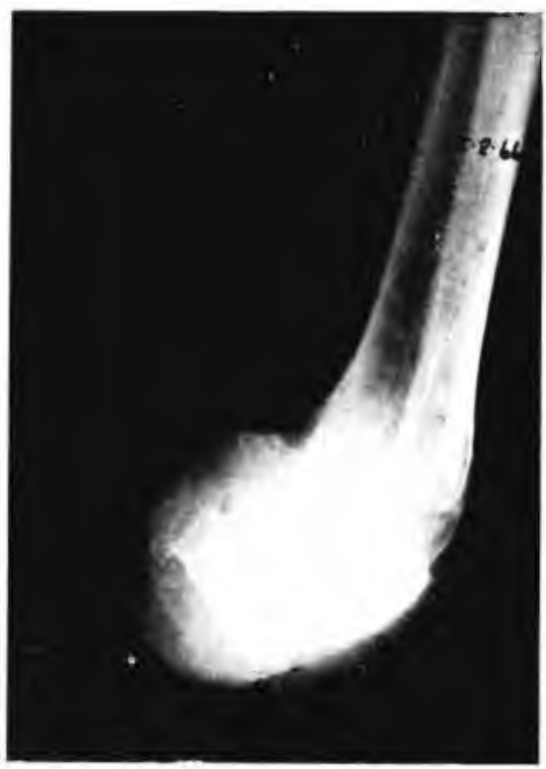

Fig. 11. Case 4: S.K. The equinus of the left foot is clearly seen, as is the fact that the pressure bearing area is now over the anterior end of the calcaneus.
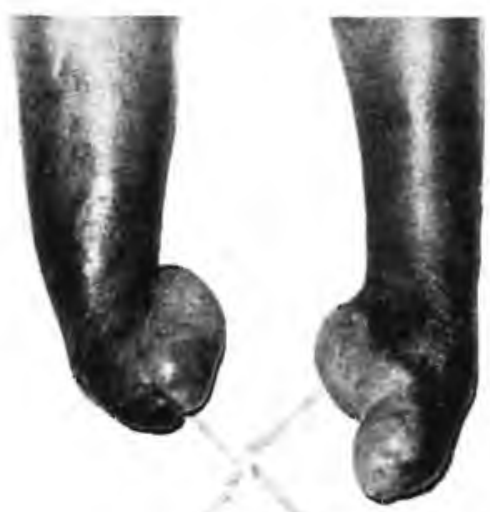

Fig. 12. Case $5:$ N.W., Leper. Note the gross equinus deformity on the right, and equino-varus on the left.

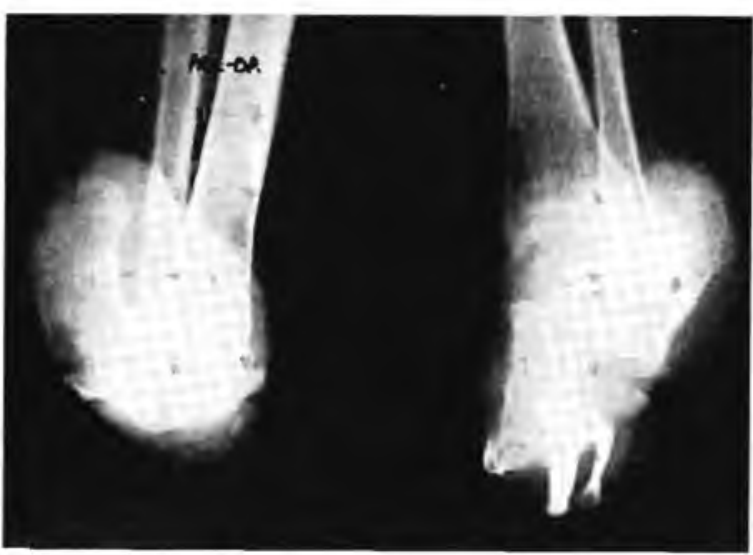

Fig. 13. Case 5: N.W. Radiographic appearance of feet in Fig. 12. 